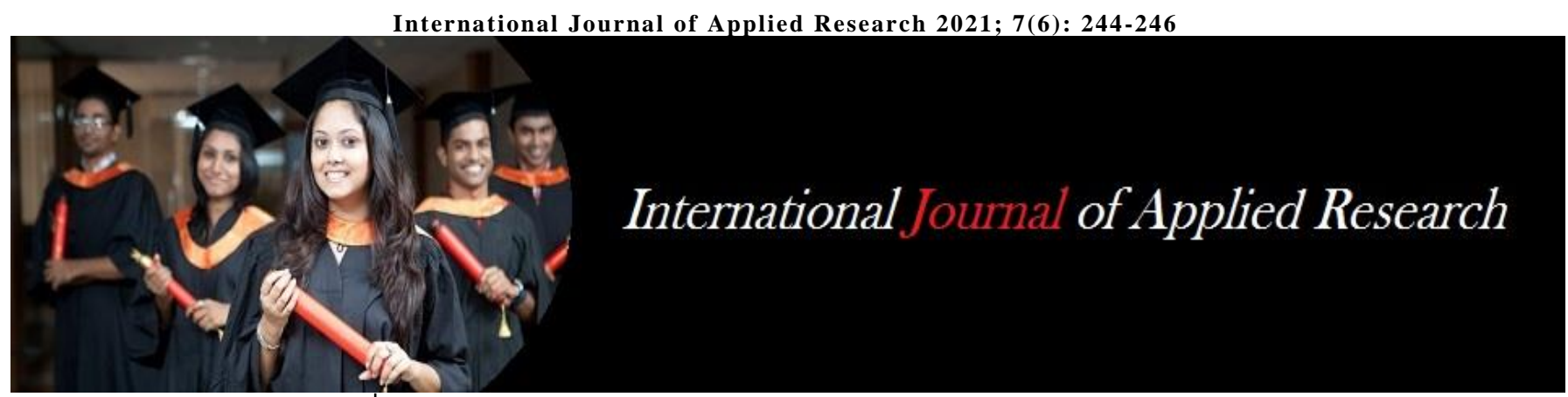

ISSN Print: 2394-7500 ISSN Online: 2394-5869 Impact Factor: 8.4

IJAR 2021; 7(6): 244-246 www.allresearchjournal.com

Received: 16-04-2021 Accepted: 18-05-2021

Garima Chaudhary Child Health Nursing Department, Rufaida College of Nursing, Jamia Hamdard, India

Jahanara Rahman

Assistant Professor, Child

Health Nursing Department, Rufaida College of Nursing,

Jamia Hamdard, India
Corresponding Author: Garima Chaudhary Child Health Nursing Department, Rufaida College of Nursing, Jamia Hamdard, India

\section{Care of child having bladder exstrophy and epispadias}

\section{Garima Chaudhary and Jahanara Rahman}

DOI: $\underline{\text { https://doi.org/10.22271/allresearch.2021.v7.i6d.8693 }}$

\section{Abstract}

The bladder exstrophy-epispadias-cloacal exstrophy complex is caused by a developmental abnormality that occurs 4-5 weeks after conception in which the cloacal membrane is not replaced by tissue that will form the abdominal muscles. The treatment includes Bladder closure and abdominal wall repair operation, Kelly procedure, Bladder Augmentation, and Epispadias repair.

Keywords: bladder exstrophy, epispadias

\section{Introduction}

The three distinct anomalies which constitute the exstrophy-epispadias complex are believed to represent a spectrum of abnormalities arising from failure of development of the lower abdominal wall during the early weeks of gestation ${ }^{[1]}$.

\section{Bladder Exstrophy}

Failure of migration of mesoderm to the area of the cloacal membrane, and subsequent lack of development of an intermediate layer between its inner (endodermal) and outer (ectodermal) layers, leads to rupture and exposure of an open bladder plate and urethra ${ }^{[1]}$.

\section{Cloacal Exstrophy}

Cloacal exstrophy is a more severe variant in which there is probably an additional failure of septation of the cloaca, so that the bladder anteriorly is not separated from the hindgut posteriorly. This has the effect of bringing both bladder and bowel components to the surface, with a central bowel field that lies in the midline between two separated halves of the bladder ${ }^{[1]}$.

\section{Primary Epispadias}

In primary epispadias the urethra opens on the dorsal aspect of the penis. Depending on the severity of the epispadias, the urethra may open distally on the glans (glanular epispadias), on the shaft ${ }^{[1]}$.

\section{Types}

\subsection{Classic Bladder Exstrophy}

Classic Bladder Exstrophy presents at birth with a visible bladder plate below a low-set umbilical cord. The mucosa may become quite inflamed and polypoid, especially after a few hours' exposure following delivery. The shaft of the penis is usually short and thick with a good-sized glans. Although the genitalia are usually recognisably male or female, there may be some confusion over gender in the referring hospital ${ }^{[1]}$.

\subsection{Cloacal Exstrophy}

Cloacal exstrophy is a severe birth defect in which there is usually a membrane-covered area on the abdominal wall that contains the abdominal contents (omphalocele). The bladder is divided in two halves and males have a penis split in two halves. Females have a clitoris divided in two halves and may have two vaginal openings. The opening of the rectum to the outside of the body is usually missing or abnormally small ${ }^{[2]}$. 


\section{Incidence}

The birth prevalence of classic bladder exstrophy has been estimated to be between 1 in 10,000; and 1 in 50,000 live births. Males are affected 2-3 times more often than females. Isolated epispadias occurs in approximately 1 in 112,000 live male births and 1 in 400,000 live female births. Cloacal exstrophy occurs in approximately 1 in 4,00,000 live births ${ }^{[2]}$.

\section{Cause}

The bladder exstrophy-epispadias-cloacal exstrophy complex is caused by a developmental abnormality that occurs 4-5 weeks after conception in which the cloacal membrane is not replaced by tissue that will form the abdominal muscles ${ }^{[2]}$.

\section{Diagnosis}

Prenatal ultrasound examination of a fetus with the complex may reveal absence of bladder filling, low-set umbilical cord, separation of pubic bones, small genitals and an abdominal mass that increases in size as the pregnancy progresses ${ }^{[2]}$.

\section{Clinical Manifestations}

- Bladder mucosa is red at birth, and mucosal polyps may be seen.

- Instead of its normal round shape, the bladder is flat.

- Inflammatory changes of whitish coating, ulcerations and hyperplastic formations (If closure is delayed)

- Thin skin stripes mark transition between normal skin and squamous metaplastic area

- Low placed or absent umblicus

- Wide separation of pubic symphysis with separation of rectus abdominis muscle.

- Genitalia may be ambiguous

- Most patients have bilateral inguinal hernias

- "Waddle" gait

\section{Management}

\subsection{Bladder closure and abdominal wall repair} operation - first few days after birth

This operation closes the bladder and abdominal wall, so that the bladder is inside the body and in the correct position. After the operation, urine will drain from the bladder through a number of catheters (plastic tubes) placed in the bladder.

For the first day or so, they will have an intravenous infusion (drip) giving fluids and medications until the bladder starts to recover. The child will need to have regular pain relief after the operation. Initially, pain relief will be given through an epidural.

They may also have ureteric stents in place, which are thin tubes inserted through the abdomen into the child's bladder and up each ureter. These drain away urine while the bladder recovers from surgery. A urethral stent will also have been inserted into the urethra to keep it open while the area heals.

After the first week, some of the tubes will be removed. The drip will be removed when the child starts feeding again. The epidural is usually removed three to five days after the operation. Seven days after the operation, the ureteric stents will be removed if they were inserted. Around three months later, the child will have a cystoscopy to check how the bladder is healing ${ }^{[3]}$.

\subsection{Kelly procedure - at one to two years old}

After the initial closure of the bladder exstrophy, there is no sphincter at the junction of the bladder and urethra. The Kelly procedure (also called a soft tissue reconstruction of the bladder neck) uses existing muscle and soft tissue to create a ring of muscle that acts like a sphincter. This holds urine in the bladder allowing it to stretch and gain more capacity and also helps form a strong stream of urine when weeing.

The surgeon can create a tummy button (omphaloplasty) during the same operation if preferred.

In boys, the Kelly procedure may also involve a reconstruction of the urethra and penis, or it may be done in a separate operation at a later stage.

During the same operation, the ureters may be re-positioned within the bladder if they are not joining the bladder in the correct place. This can cause a condition called vesicoureteric reflux (VUR) where the valves can fail, allowing urine to flow backwards from the bladder towards the kidney. Depending on the severity of the VUR, sometimes the urine can flow backwards as far as the kidneys. This can damage the kidney and eventually lead to kidney failure. The ureteric re-implantation operation involves disconnecting the ureters and re-attaching them in the correct place ${ }^{[3]}$.

\subsection{Bladder Augmentation}

Following the Kelly procedure, if the ring of muscle around the base of the bladder is too weak, urine could dribble out all the time. This also happens if the bladder cannot hold enough urine. This can be improved with an operation called bladder augmentation, which involves making the bladder larger, and therefore able to hold a larger volume of urine, using a section of intestine. These children then empty their bladder using a catheter to drain away the urine. The catheter can be inserted either into the urethra or a specially made channel called a Mitrofanoff ${ }^{[3]}$.

\subsection{Epispadias Repair}

The Cantwell-Ransley epispadias repair is favoured by many surgeons and can be performed in the first year of life. In this procedure the urethral plate is fully mobilised off the penile corpora from the proximal urinary outlet and corporeal bodies but is left attached to the glans. The urethral plate is then tubularised and brought to a ventral position, below the corpora cavernosa, which are then approximated dorsal to the urethra ${ }^{[1]}$.

The majority of girls with epispadias are incontinent, reconstructive surgery must be directed to correction of the incontinence as well as the genital abnormality. The Kelly procedure offers good prospects of continence coupled with an improved external cosmetic appearance ${ }^{[1]}$.

\section{Prognosis}

Urinary control most often happens after the neck of the bladder is repaired. This surgery is not always successful. The child may need to repeat the surgery later on. Even with repeat surgery, a few children will not have control of their urine. They may need catheterization.

\section{Nursing Diagnosis \\ 12.1 Pre-Operative}

1. Impaired Skin Integrity related to exposed urinary bladder along with bifid glans and scrotum. 
2. Fluid Volume Deficit related to increased insensitive water loss in Exstrophy from exposed viscera.

3. Altered Family Process related to Congenital Defects.

4. Anxiety related to disease process and surgical management.

\subsection{Post-Operative}

1. Discomfort related to pain at surgical site.

2. Fluid Volume Deficit related to decreased oral intake of feeds secondary to sedation following surgery.

3. Impaired skin integrity related to surgical intervention.

4. Risk for surgical site infection related to invasive lines and procedures.

5. Disturbed body image related to surgical management as evidenced by sutures, urinary draining tubes and collection devices.

6. Risk for complications (Urinary or surgical site infections, Fistulae in the suprapubic or penile incisions) related to surgical management. ${ }^{4}$

\section{References}

1. Thomas DFM, Duffy PG. Essentials of Paediatric Urology. $2^{\text {nd }}$ edition Informa UK Ltd. 199-211.

2. Bladder exstrophy-epispadias-cloacal exstrophy complex - NORD (national organization for rare disorders) [Internet]. Rarediseases.org. 2015 [cited 2021 May 5]. Available from: https://rarediseases.org/rare-diseases/bladder-exstrophyepispadias-cloacal-exstrophy-complex

3. Frimberger D, Gearhart JP. Bladder Exstrophy and Epispadias. In: Springer Surgery Atlas Series. Berlin/Heidelberg: Springer-Verlag 2005. 589-606.

4. TM Assuma Beevi. Pediatric Nursing Care Plans (Kindle Location 1260). Kindle Edition. 